\title{
Broadband plasmonic absorber for photonic integrated circuits
}

\author{
Xiao Xiong, ${ }^{1}$ Chang-Ling Zou, ${ }^{1,}$ (a) Xi-Feng Ren, ${ }^{1, b}$ ) and Guang-Can Guo ${ }^{1}$ \\ Key Lab of Quantum Information, University of Science and Technology of China, Hefei 230026, \\ P. R. China
}

(Dated: 10 October 2018)

The loss of surface plasmon polaritons has long been considered as a fatal shortcoming in information transport. Here we propose a plasmonic absorber utilizing this "shortcoming" to absorb the stray light in photonic integrated circuits (PICs). Based on adiabatic mode evolution, its performance is insensitive to incident wavelength with bandwidth larger than $300 \mathrm{~nm}$, and robust against surrounding environment and temperature. Besides, the use of metal enables it to be very compact and beneficial to thermal dissipation. With this $40 \mu \mathrm{m}$-long absorber, the absorption efficiency can be over $99.8 \%$ at $1550 \mathrm{~nm}$, with both the reflectivity and transmittance of incident light reduced to less than $0.1 \%$. Such device may find various applications in PICs, to eliminate the residual strong pump laser or stray light.

Strong light is widely used in optical circuits: pumping the active medium for light amplification and for coherent laser emission or the quantum dot for single photon source, pumping nonlinear optical processes such as fourwave mixing $\frac{1}{}$, and actuating and controlling the microand nano-mechanical oscillators 2.3 . On the other hand, in photonic integrated circuits (PICs), weak signal light or single photon are used for classical and quantum information processing. Therefore, stray light from strong pump/control light will induce errors in information processing and high noise background. That is to say, an optical "dump", which can perfectly absorb the strong pump/control light with no reflection, is quite necessary in PICs. During past decades, a variety of spatial two-dimensional (2D) or three-dimensional (3D) absorbers composed of array of plasmonic nano-resonators have been proposed $\underline{4}^{-\underline{9}}$. Surprisingly, however, integrated absorber compatible with dielectric waveguide has been omitted.

One may borrow the idea from the spatial absorbers to PICs by using critically coupled resonators to absorb the incoming light with neither reflection nor transmission. However, there exists several limitations in resonatorbased absorber. Firstly, the cavity has to work under the critical coupling condition, which is highly dependent on the geometric parameters of cavity. Secondly, the absorption spectrum is a Lorentz-shaped profile with a relatively narrow bandwidth. Thirdly, the resonance wavelength is sensitive to temperature, thus the absorption spectrum will shift due to light heating, and lead to instability. Fourthly, finite cavity resonance also corresponds to limited response speed, which means laser pulse can not be absorbed effectively before stable field is established in the cavity. In addition, the resonances are polarized in PICs, hence the two orthogonal polarizations usually cannot be absorbed simultaneously.

In this Letter, we propose a broadband plasmonic absorber for PICs. Since surface plasmon polaritons (SPPs)

\footnotetext{
a) Electronic mail: clzou321@ustc.edu.cn

b) Electronic mail: renxf@ustc.edu.cn
}

enable extreme confinement and have big inherent losses in propagation 10.11 , they are of course the best candidate to fulfill the task for absorption in PICs. However, the impedance mismatch between dielectric and plasmonic modes will inevitably lead to reflection. Therefore, we utilize the adiabatic mode evolution to efficiently convert the dielectric waveguide mode to plasmonic mode $\frac{12}{2}$. As a result, the absorption can be achieved over a wide range of wavelength, and remains robust against surrounding environment and temperature ${ }^{13}$. By a simple circular arc structure of dozens of microns long, we realized a waveguide-integrated adiabatic absorber, whose absorption efficiency can reach more than $99.8 \%$ at $1550 \mathrm{~nm}$, with both the reflectance and transmittance reduced to less than $0.1 \%$. And the absorption efficiency keeps over 99.5\% from $1400 \mathrm{~nm}$ to $1700 \mathrm{~nm}$ for both horizontal $(H)$ and vertical $(V)$ polarizations. Additional to the excellent optical performance, the absorber has very good thermal conductivity considering that laser heating can be conducted by metal wires or thermal antenna. We believe this compact, broadband, robust and thermalconductive absorber may find applications in practical PICs.

The schematic illustration of our proposal is shown in Fig. 1(a). A silicon waveguide is fabricated on silica substrate, with the waveguide cross-section being $300 \mathrm{~nm} \times 400 \mathrm{~nm}$. And gold (Au) of height $h$ covers the end of the waveguide and consists of two parts: the tangent circular arc with radius of curvature being $r$, and the rectangle with width $w$ and length $l$. The working wavelength is focused on the telecommunication band around $\lambda=1550 \mathrm{~nm}$. The refractive indices of silicon and silica are $n_{S i}=3.5$ and $n_{\mathrm{SiO}_{2}}=1.444$, respectively. And the permittivity of $\mathrm{Au}$ is calculated with Lorentz-Drude model according to parameters from Ref ${ }^{14}$. Then we performed numerical simulations with COMSOL Multiphysics 4.3 to test the performance of this absorber. It's obvious that the performance of an absorber should be estimated with two parameters: transmittance $T$ and reflectivity $R$. Here, $T$ is defined as the ratio of total outgoing energy flux to total incident energy flux ${ }^{15}$, and $R$ is the ratio of backward-propagating intensity to forward- 


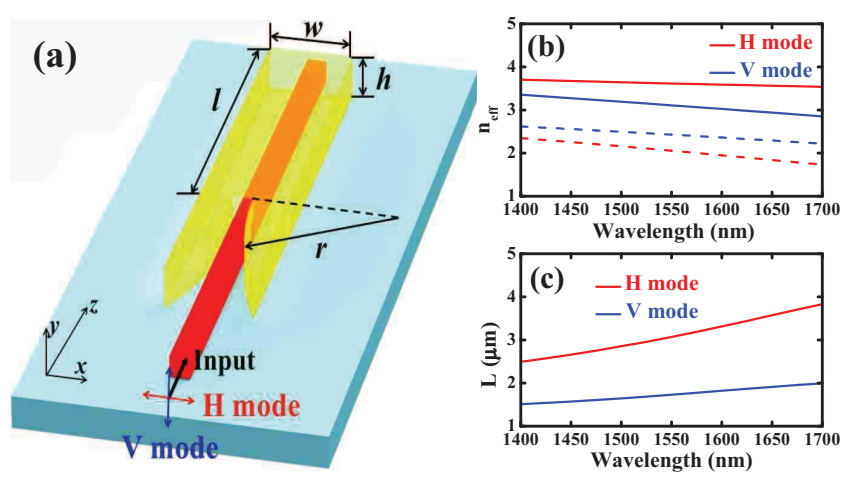

FIG. 1. (a) Schematic of the adiabatic absorber, which consists of the adiabatic circular arc (radius $r$ ) and the absorption rectangle $($ size $l \times w \times h)$. Incident light includes two orthogonal polarizations ( $H$ and $V$ modes) and propagates along the $z$-axis. (b) Dependence of effective refractive index $n_{\text {eff }}$ on incident wavelength $\lambda$ for dielectric $H / V$ modes (dashed red/blue curves) and plasmon $H / V$ modes (solid red/blue curves), respectively. Here, $l=20 \mu \mathrm{m}, w=1.5 \mu \mathrm{m}$, $h=0.8 \mu \mathrm{m}$. (c) Dependence of propagation length $L$ on $\lambda$ for plasmon $H$ (red curve) and $V$ (blue curve) modes, with $w=1.5 \mu \mathrm{m}$ and $h=0.8 \mu \mathrm{m}$.

propagating intensity.

Firstly, we calculated the effective refractive index $n_{\text {eff }}$ of dielectric eigenmode (in pure silicon waveguide without metal) and plasmon eigenmode (in metal-covered silicon waveguide) as a function of wavelength $\lambda$, as shown in Fig. 1(b). We observed that the difference between dielectric and plasmon modes is rather big for both polarizations, especially for $H$ polarization. This big difference, which indicates the mode mismatching between dielectric and plasmon modes, will induce large reflectivity on the interface between pure silicon waveguide and metal-covered silicon waveguide. In order to reduce the reflectivity, a designed taper can be used to convert dielectric guided modes to plasmon modes adiabatically 16,17. Here, we just adopted a circular arc which is quite simple but works well.

Then, we calculated the propagation length of plasmon modes $L$ depending on $\lambda$ for $H$ and $V$ modes, respectively, as displayed in Fig. 1(c). As the incident wavelength increases, the propagation length $L$ increases for both polarizations. And the propagation length $L$ of $H$ mode keeps larger than that of $V$ mode. Nevertheless, for a wide bandwidth of $300 \mathrm{~nm}$, the propagation length $L$ of both $H$ and $V$ modes are below $4 \mu \mathrm{m}$. According to $I(l)=I_{0} e^{-l / L}$, we get to know that longer $l$ will bring better absorption performance. Especially, at $\lambda=1550 \mathrm{~nm}$, the propagation length of $H$ mode is larger, being $L=3 \mu \mathrm{m}$. Thus, we fixed $l$ to be $l=20 \mu \mathrm{m}$, expecting an absorption efficiency of $I(l) / I_{0}=e^{-20 / 3} \approx 0.1 \%$. In addition, we also calculated the dependence of propagation length $L$ on $w$ and $h$ for $H$ and $V$ modes. It increases with increasing $w$ and $h$ firstly, and get saturated over certain value $(w>0.5 \mu \mathrm{m}$,

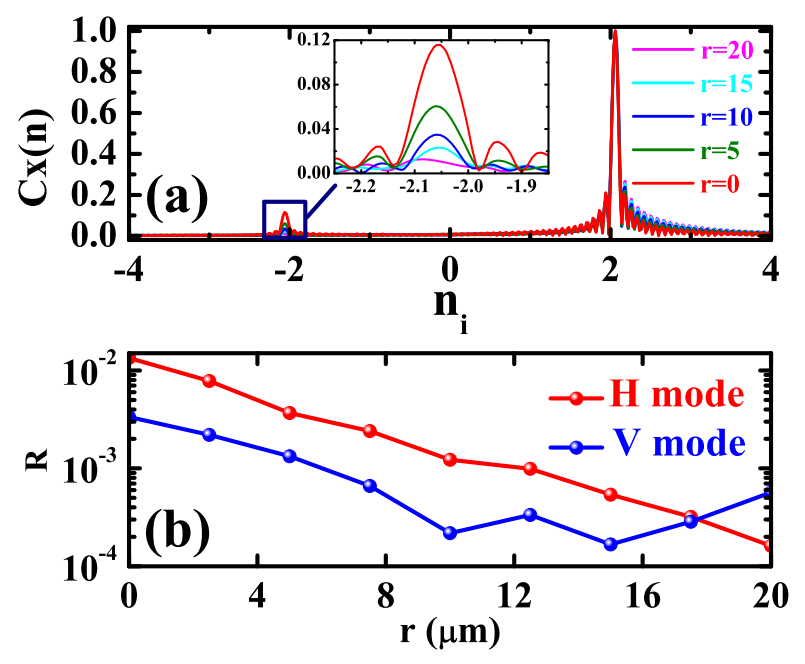

FIG. 2. (a) Fourier-transformed spectrum of electric field component $E_{m}(z)$ in the adiabatic part, with $r=0,5,10$, 15, 20, respectively. Here, the incident field is $H$ mode, with $l=20, w=1.5, h=0.8$ (in the unit of $\mu \mathrm{m}$ ). Inset: enlarged view of the peak which stands for the reflected field. (b) Dependence of reflectivity $R$ on $r$ for $H$ and $V$ modes, respectively.

$h>0.5 \mu \mathrm{m})$. This is because energy is mostly confined in the metal when the Au coating is ultra-thin, rather than in the waveguide. When $w>0.5 \mu \mathrm{m}$ and $h>0.5 \mu \mathrm{m}$, the energy of propagating mode is mostly confined in the dielectric waveguide, then the absorption of this device won't be sensitive to the size of metal coating. So we fixed $w=1.5 \mu \mathrm{m}$ and $h=0.8 \mu \mathrm{m}$ in the saturated region in our studies.

It is conceivable that if the conversion from dielectric mode to plasmon mode in the first half of this absorber is more adiabatic, the reflectivity due to momentum mismatching will be smaller ${ }^{12}$. Similarly, if the plasmon mode propagates farther, the loss of incident light due to metallic absorption is larger. That is to say, larger $r$ brings smaller $R$ while longer $l$ brings smaller $T$. Next, we'll confirm our predictions with 3D numerical simulations. Matched fundamental modes with two orthogonal polarizations ( $H$ and $V$ modes) are incident at the waveguide facet, respectively, and propagate along $z$ direction, with the coordinate origin located at the waveguide center of incident plane. Note that, benefiting from the propagation losses in metal, $T$ can always be reduced as small as possible, as long as $l$ is large enough. As a result, we focus on the influence of adiabatic radius $r$ on reflectivity $R$ in the following.

Denote the electric field component along $m$ direction ( $m=x(y)$ for $H(V)$ polarization) as $E_{m}(z)$, which is distributed along the center line of waveguide as a function of $z$. In the adiabatic part, $E_{m}(z)$ should be a sum of forward- and backward-propagating dielectric modes as 


$$
E_{m}(z)=\sum_{n_{i}} C_{m}\left(n_{i}\right) e^{-j n_{i} k z},
$$

where $n_{i}$ is the effective refractive index of different eigenmodes, $C_{m}\left(n_{i}\right)$ is the corresponding mode amplitude, and $k=\frac{2 \pi}{\lambda}$ is the wave number. By applying Discrete Fourier Transform (DFT) to $E_{m}(z)$, the coefficient $C_{m}\left(n_{i}\right)$ can be expressed as

$$
C_{m}\left(n_{i}\right)=\frac{k}{2 \pi} \sum_{z} E_{m}(z) e^{j n_{i} k z} .
$$

And the sign before $n_{i}$ represents exactly the energy propagation direction. As shown in Fig. 2(a), the Fourier-transformed spectrum consists of two main peaks that are symmetric distributed. The right main peak stands for the forward-propagating incident mode with $n=+n_{i}$, while the left one is the backward-propagating reflected mode with $n=-n_{i}$. Here, the priminent peak for $n<0$ corresponds to reflected fundamental mode. Then, the reflectivity $R$ is obtained as

$$
R=\left|\frac{C_{m}\left(-n_{i}\right)}{C_{m}\left(+n_{i}\right)}\right|^{2} .
$$

Here, the incident field has been normalized to be unit for simplicity. Besides, it's worth noting that there are many side lobes around the main peaks, which originates from the finite sampling interval. Furthermore, the dependences of $R$ on the arc radius $r$ for different polarization are plotted in Fig. 2(b). As we can see, for $r>15: \mu \mathrm{m}$, the reflectivity $R$ are reduced to be less than $0.1 \%$ for both $H$ and $V$ modes.

Similarly, by applying DFT to $E_{m}(z)$ in the absorption part, we obtained the Fourier-transformed spectra of $H$ and $V$ modes, which contain the information about plasmon eigenmodes. As illustrated in Fig. 3(a), the normalized spectrum for $H$-polarized incident field consists of two peaks. While no peak appears in the region $n_{i}<0$, it means that there are no reflected modes in the absorption part of this absorber. As for the effective refractive indices corresponding to the two peaks, they are exactly consistent with the $H$-polarized plasmon eigenmodes calculated under 2D simulations (shown in the inset in Fig. $3(\mathrm{a})$ ), with the major (minor) peaks being plasmon fundamental (high-order) eigenmode. Unlike the situation in Fig. 3(a), the normalized spectrum for $V$-polarized incident field (Fig. 3(b)) includes four peaks, and they stand for the four $V$-polarized plasmon eigenmodes of different order (inset of Fig. 3(b)), respectively. Here, many $V$-polarized plasmon eigenmodes of higher-order are excited, due to the discontinuity of metal in the interface between the arc and rectangle at the top of waveguide for $V$ polarization. This sudden change of refractive index also results in the special trend of the blue curve shown in Fig. 2(b), where $R$ of $V$ mode does not decrease monotonously with the increase of $r$. While for $H$
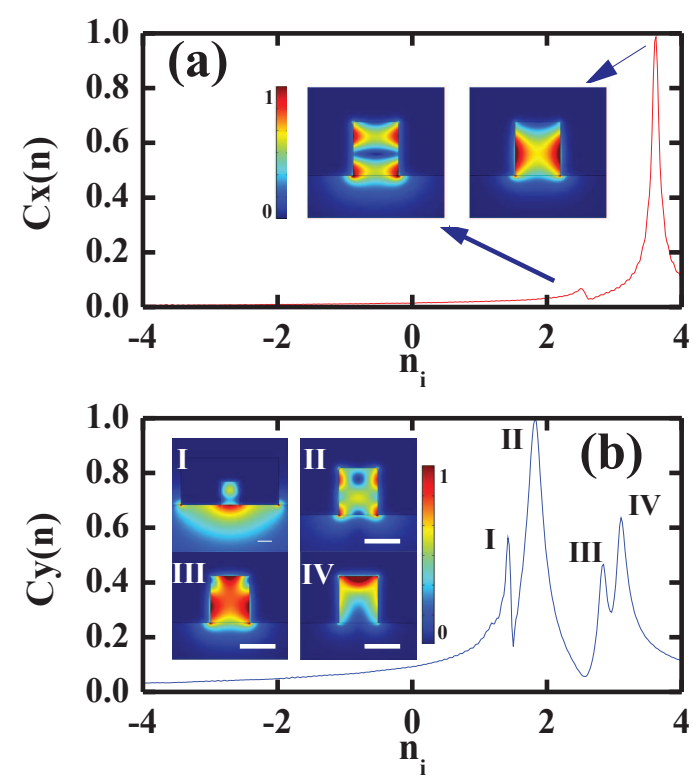

FIG. 3. Normalized Fourier-transformed spectra of electric field component $E_{m}(z)$ in the absorption part for (a) $H$ mode and (b) $V$ mode, respectively, with $r=20 \mu \mathrm{m}$. Inset: the field distributions of plasmon eigenmodes, which correspond to the peaks in Fourier-transformed spectra, respectively. Scale bar in the insets I-IV: $300 \mathrm{~nm}$.
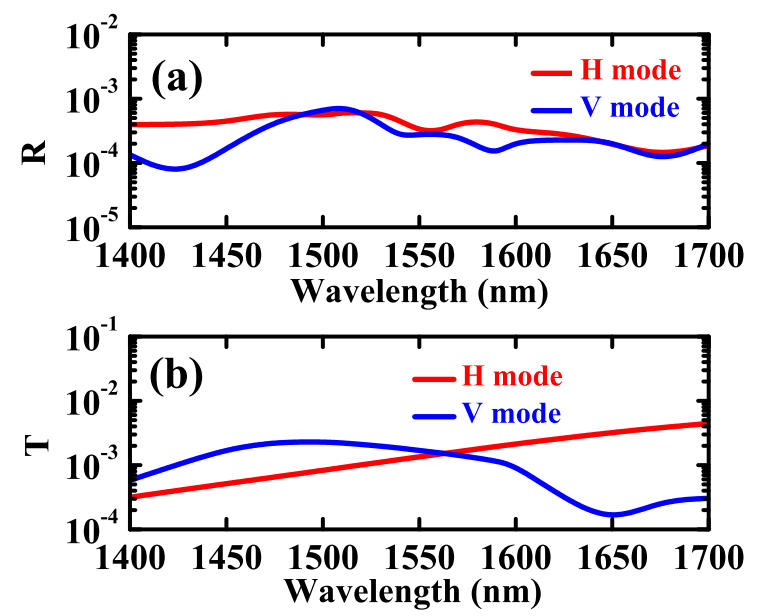

FIG. 4. Dependence of (a) $R$ and (b) $T$ on $\lambda$ for $H$ and $V$ modes. Here, $l=20, w=1.5, h=0.8, r=17.5$ (in the unit of $\mu \mathrm{m})$.

polarization, the fundamental dielectric mode is adiabatically converted into fundamental plasmon mode (with the high-order plasmon mode being negligible), and the reflectivity $R$ drops monotonously as $r$ increases, since the metal is getting close to the waveguide slowly enough. Therefore, the performance of $V$ mode can be improved by etching or evaporating a metal slope in the interface between the arc and rectangle at the top of waveguide.

Finally, we calculated the dependence of $R$ and $T$ on incident wavelength $\lambda$ for both $H$ and $V$ polarizations. 
As shown in Fig. 4(a), the reflectivity $R$ is kept less than $0.1 \%$ for both polarizations over a bandwidth as wide as $300 \mathrm{~nm}$. As for the transmission $T$ shown in Fig. 4(b), it increases with increasing incident wavelength for $H$ polarization, while maintains well below $0.3 \%$ over a bandwidth of $300 \mathrm{~nm}$ for $V$ polarization. In order to further increase the absorption efficiency, the absorption part can be engineered to be longer, which is quite convenient. Or, the waveguide in the absorption part can be tapered to adiabatically transfer more energy into the metal and improve the absorption loss.

There are still several points that we want to discuss. For instance, to further improve the absorber's performance with less reflectivity as well as less transmission, the process of mode conversion can be engineered to be more adiabatic (larger $r$ ), and the distance for absorption needs to be longer (larger $l$ ). And considering the adoption of metal, which is a good thermal conductor, heat dissipation can be easily improved by adding more metal, etching nanostructures at the top of the metal rectangle like heat sink, and guiding the thermal energy with a metallic wire or thermal emitter ${ }^{18}$. Since incident optical energy is totally converted to thermal energy, it fundamentally eliminates the negative effect of stray light. While for resonance-based absorber, a portion of the incident light will be transformed to scattering light. Additionally, since the imaginary part of the refractive index of $\mathrm{Au}$ increases with higher temperature, the increase of temperature in metal caused by energy absorption will further improve the absorption efficiency of this absorber, rather than wrecking it. Finally, since this absorber works based on adiabatic mode conversion, its performance is insensitive to geometric parameters and imposes little requirement on processing technology. Our calculation proves that, even if the lateral displacement between dielectric waveguide and plasmonic absorber is as large as $100 \mathrm{~nm}$, the absorption efficiency for both $H$ and $V$ modes can still be maintained around $99 \%$.

In conclusion, we propose a plasmonic absorber for guided waves in PICs, which is based on adiabatic mode conversion and can achieve an absorption efficiency more than $99.8 \%$ (with both reflectivity and transmission less than $0.1 \%$ ). And the use of metal enables the device to be very compact $(1 \mu \mathrm{m} \times 1 \mu \mathrm{m} \times 40 \mu \mathrm{m})$ and conducive to heat dissipation. Besides, since the absorption is realized adiabatically with a circular arc, this absorber has a bandwidth as wide as $300 \mathrm{~nm}$ for both $H$ and $V$ polarizations, and its performance is also robust against surrounding environment and temperature. It is essential for integrated photonic chips, and will find applications in PICs-based optics.

\section{ACKNOWLEDGMENTS}

This work was funded by NBRP (grant nos. 2011CBA00200 and 2011CB921200), the Innovation Funds from the Chinese Academy of Sciences (grant no. 60921091), NNSF (grant nos. 10904137, 10934006 and 11374289), the Fundamental Research Funds for the Central Universities (grant no. WK2470000005), and NCET.

${ }^{1}$ R. W. Boyd, Nonlinear optics, (Academic, 2008).

${ }^{2}$ M. Li, W. H. P. Pernice, C. Xiong, T. Baehr-Jones, M. Hochberg, and H. X. Tang, Nature 456, 480-484 (2008).

${ }^{3}$ X. Guo, C. L. Zou, X. F. Ren, F. W. Sun, and G. C. Guo, Appl. Phys. Lett. 101, 071114 (2012).

${ }^{4}$ N. Liu, M. Mesch, T. Weiss, M. Hentschel, and H. Giessen, Nano Lett. 10, 2342-2348 (2010).

${ }^{5}$ N. I. Landy, S. Sajuyigbe, J. J. Mock, D. R. Smith, and W. J. Padilla, Phys. Rev. Lett. 100, 207402 (2008).

${ }^{6}$ J. M. Hao, J. Wang, X. L. Liu, W. J. Padilla, L. Zhou, and M. Qiu, Appl. Phys. Lett. 96, 251104 (2010).

${ }^{7}$ J. Wang, Y. T. Chen, J. M. Hao, M. Yan, and M. Qiu, J. Appl. Phys. 109, 074510 (2011).

${ }^{8}$ Y. R. He, H. X. Deng, X. Y. Jiao, S. L. He, J. Gao, and X. D. Yang, Opt. Lett. 38, 1179-1181 (2013).

${ }^{9}$ S. W. Dai, D. Zhao, Q. Li, and M. Qiu, Opt. Express 21, 1312513133 (2013).

${ }^{10}$ P. G. Kik and M. L. Brongersma, Surface plasmon nanophotonics, (Springer, 2007).

${ }^{11} \mathrm{D}$. Sarid and W. Challener, Modern introduction to surface plasmons: theory, mathematica modeling and applications (Cambridge, 2010).

${ }^{12}$ C. L. Zou, F. W. Sun, C. H. Dong, Y. F. Xiao, X. F. Ren, L. Lv, X. D. Chen, J. M. Cui, Z. F. Han, and G. C. Guo, IEEE Photonic Tech. L 24, 434-436 (2012).

${ }^{13}$ C. H. Dong, C. L. Zou, X. F. Ren, G. C. Guo, and F. W. Sun, Appl. Phys. Lett. 100, 041104 (2012).

${ }^{14}$ A. D. Rakic, A. B. Djurisic, J. M. Elazar, and M. L. Majewski, Appl. Opt. 37, 5271-5283 (1998).

${ }^{15}$ S. S. Wang, Z. F. Hu, H. K. Yu, W. Fang, M. Qiu, and L. M. Tong, Opt. Express 17, 10881-10886 (2009).

${ }^{16}$ Y. N. Zhang, C. L. Li, and M. Loncar, Opt. Lett. 38, 646-648 (2013).

${ }^{17}$ P. Ginzburg, D. Arbel, and M. Orenstein, Opt. Lett. 31, 32883290 (2006)

${ }^{18}$ J. A. Schuller, T. Taubner, and M. L. Brongersma, Nat. Photonics 3, 658-661 (2009). 